\title{
OBSERVATION OF BUNCH MOTION DUE TO THE LONGITUDINAL DIPOLE-COUPLED BUNCH INSTABILITY AT CESR*
}

\author{
R. Holtzapple ${ }^{\#}$, M. Billing, Cornell University, Ithaca, New York
}

\begin{abstract}
In the past a longitudinal dipole-coupled bunch instability had limited high current operation at CESR and resulted in a degradation of luminosity performance. A longitudinal feedback system successfully damps this instability and the exchange of superconducting RF cavities for normal conducting RF cavities in CESR has further reduced the instability's strength. In this paper measurements of the longitudinal dynamics using a dualaxis synchroscan streak camera are presented. The measurements were made on single trains of bunches and give a characterization of the instabilities and modes of oscillation.
\end{abstract}

\section{INTRODUCTION}

To achieve high luminosity, CESR is operated with multiple bunches. Under present conditions, there are up to nine trains of bunches, with up to five bunches in each train, for a maximum of 45 positron and electron bunches. One limitation to high luminosity is a longitudinal dipolecoupled bunch instability (LDCBI) which occurs when multiple bunches are present. The LDCBI was first noted during operation with nine trains of two bunches in CESR. At the instability threshold, a rapid growth of the spectral lines at the synchrotron sidebands of the rotation harmonics was observed. The growth of amplitude for the spectral lines depends upon the spacing of the trains and bunches and it is the indication that the instability threshold is exceeded. The threshold of the LDCBI depends on the spacing between bunches and the number of bunches in each train. The LDCBI degrades the machine luminosity and, at high current, is responsible for beam loss.

To combat the LDCBI, two modifications were made: 1) a low $\mathrm{Q}, 1.1 \mathrm{GHz}$. accelerating cavity is used as a longitudinal feedback cavity to damp the LDCBI. 2) To increase the current threshold and reduce the effect of the LDCBI, four single cell niobium superconducting RF cavities have been installed in CESR replacing twenty cells of normal conducting RF cavities. The superconducting RF cavities have more strongly damped higher order modes than the previous normal conducting RF cavities[1]. Measurements of the LDCBI at CESR have been made using a dual sweep synchroscan streak camera. A previous characterization of the LDCBI were performed with a single sweep steak camera with the normal conducting RF cavities[2].

\footnotetext{
* This work was supported by the National Science Foundation. \# Present e-mail: RLH@SLAC.Stanford.edu.
}

\section{INSTABILITY MEASURMENTS}

A typical dual-sweep synchroscan streak camera image is shown in fig. 1. The vertical axis on the image is the fast streak speed and the horizontal axis is the slow streak speed. The longitudinal information of each light pulse (trains in this instance) is extracted in the following way. Each pulse is selected with the vertical cursors shown in the fig. 1. Between the vertical cursors, the pixels are summed horizontally to create the longitudinal profile of the bunch distribution (displayed on the left-hand side of the image). For each image, a longitudinal profile for each light pulse is created, saved, and processed off line. The processing includes fitting the longitudinal profile to an asymmetric Gaussian function [2].

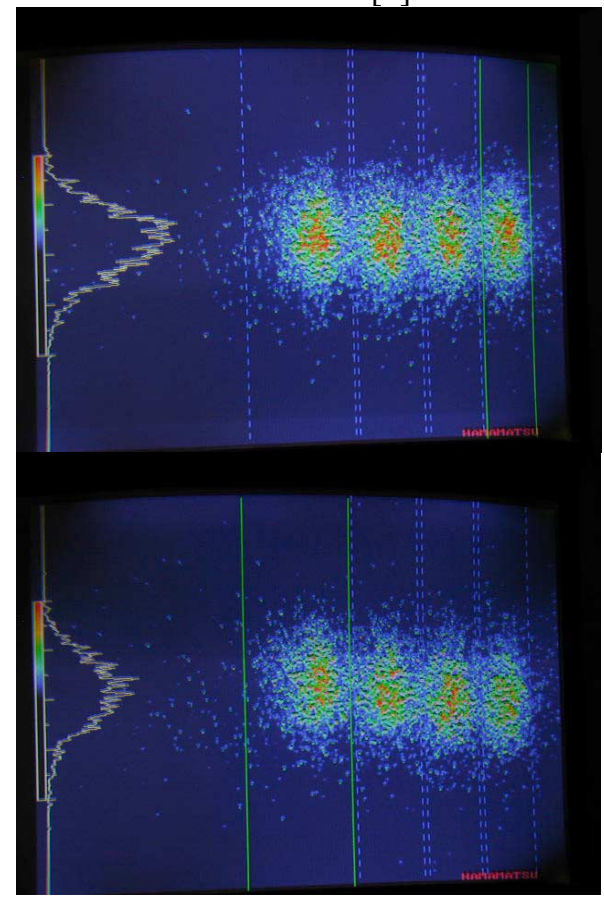

FIG. 1 (color). Typical streak camera images of a single train of bunches in CESR with the longitudinal feedback turned on and stable bunches(top) and feedback off (bottom) and unstable bunches.

The measurements were made above the LDCBI current threshold when the instability was (was not) present which occurs when the longitudinal feedback system is turned off (on). During normal operation, the feedback system must remain on. The following experiment was performed during special machine studies times when the feedback system could be turned off to induce the instability.

Setting the slow streak speed to a full sweep range of $100 \mathrm{~ns}$ allows the single train dynamics to be measured. The measurements were made with nine trains of 
positrons, with four bunches per train, present in CESR. The bunch spacing in each train was $14 n$ s. At this bunch spacing, the instability threshold is approximately $180 \mathrm{~mA}$ total or $5 \mathrm{~mA}$ per bunch. Measurements were made at total beam current between $180 \mathrm{~mA}$ and $350 \mathrm{~mA}$.

The presence of the LDCBI was determined by two methods: 1) Above the instability threshold with longitudinal feedback turned off, a signal indicating a dipole instability is observed using a spectrum analyzer. The signature of the instability is synchrotron sidebands clearly visible at $\mathrm{f}=\mathrm{nf}_{\mathrm{r}} \pm \mathrm{f}_{\mathrm{s}}$. During this experiment, a spectrum analyzer was used to measure the amplitude and frequency of the beam spectra for $\mathrm{f}=\mathrm{f}_{\mathrm{r}}+\mathrm{f}_{\mathrm{s}}($ table 1). 2) The dual-sweep synchroscan streak camera can detect the LDCBI. When the instability is present, the profiles of the four bunches do not align vertically, as shown in figure 1.
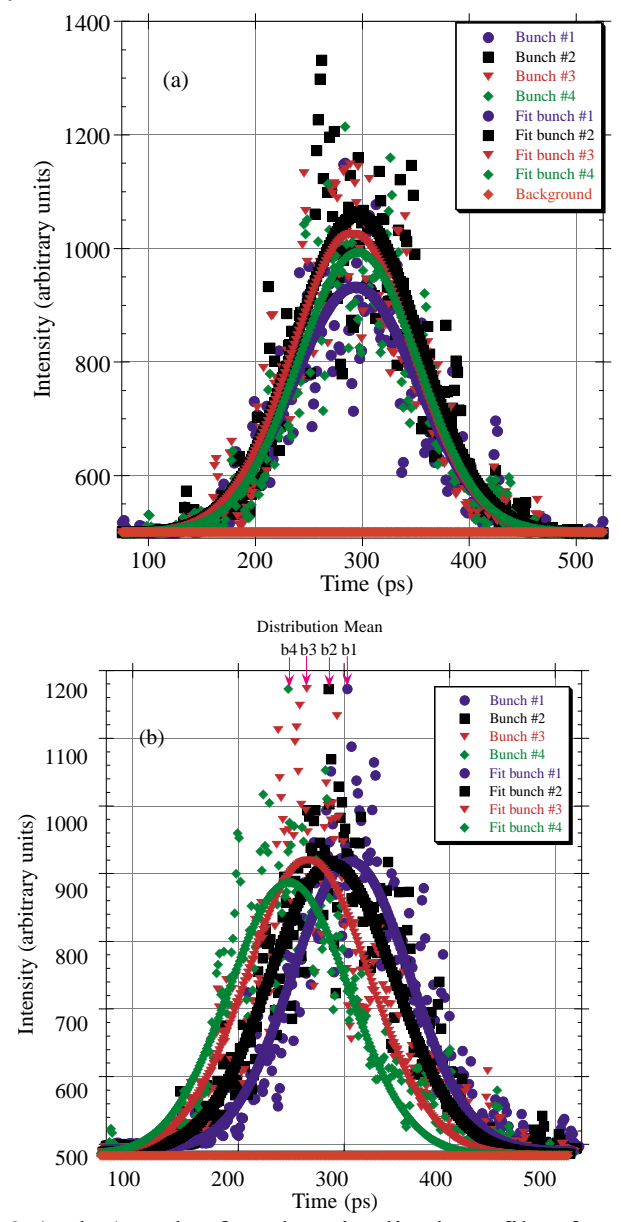

FIG. 2 (color). The four longitudinal profiles from a single image when the dipole mode coupled bunch instability is (a) not present and (b) present. The total positron current when these images were taken was $276 \mathrm{~mA}$.

Several single trains images, like the ones in fig. 1, were made at different beam currents, with and without the instability present. The images with the instability present were chosen due to observed large time oscillations. The amplitude of time oscillation for each bunch is quantified by computing the shift in its centroid (mean) relative to the other bunches in the train. If the bunches are stable, the centers of the four bunch distributions in a train should superimpose in time. If the longitudinal dipole-coupled bunch instability is present, the bunches centroids oscillate in time, and will not in general be aligned in time. In fig. 2 the longitudinal distributions of bunches in a single train are superimposed for the case when the beam is stable (a) and unstable (b).

A spectrum analyzer was used to determine the strength of the LDCBI in conjunction with the streak camera images. As the total beam current increase the amplitude of the instability increased for the first three measurements (I=183-276mA), and decreased for the last two measurements $(\mathrm{I}=326-350 \mathrm{~mA})$. This drop in amplitude can be attributed to a different mode of oscillation in the bunch train, and will be discussed later.

\begin{tabular}{|l|l|l|}
\hline Current $(\mathrm{mA})$ & $\mathrm{fr}+\mathrm{fs}(\mathrm{kHz})$ & Amplitude $(\mathrm{dBm})$ \\
\hline \hline $183-181$ & 410.75 & -35.3 \\
\hline $227-224$ & 410.68 & -19.6 \\
\hline $276-272$ & 410.60 & -18 \\
\hline $326-316$ & 410.45 & -31.1 \\
\hline $350-344$ & 410.38 & -28.8 \\
\hline
\end{tabular}
frequency, and spectral intensity for these measurements.

Under stable conditions the bunches in a train are separated by $14 \mathrm{~ns}$. When the instability is present, the bunches in a train oscillate about the $14 \mathrm{~ns}$ spacing. The amplitude of the time oscillation is determined from the longitudinal profiles fit to an asymmetric Gaussian function. The centroid position of the bunch is determined from the fit to the data. For our analysis, the amplitude of the time oscillation is referenced to the leading bunch (first bunch in the train). The change in arrival time for the bunches after the leading bunch is given by

$$
\delta \mathrm{t}_{\mathrm{n} 1}=\operatorname{Mean}_{\mathrm{n}}-\text { Mean }_{1} \text { for } \mathrm{n}=2,3,4
$$

where $\mathrm{n}$ is the trailing bunch number. The sign convention is the following: A positive $\delta t_{n 1}$ means the trailing bunch arrives $(n-1) 14 n s+\delta t_{n 1}$ after the leading bunch in a train. The time deviation from the lead bunch is displayed for a number of sample triggers in fig. 3 for each of three different cases: (a) when the train was stable at a current of $276 \mathrm{~mA}$, (b) when the train was unstable at a current of $276 \mathrm{~mA}$, and (c) when the train was unstable at a current of $326 \mathrm{~mA}$.

Several comments can be made about the results:

1) Using this analysis technique it is easy to see that the bunches in a train oscillate in time. The longitudinal feedback system stabilizes the bunch time oscillations to a few picoseconds. When turned off, the time oscillation has been measured to approximately $100 \mathrm{ps}$. The unstable time oscillations substantially degrade the High-Energy Physics luminosity.

2) The data suggests a change in bunch oscillation mode occurs between $276 \mathrm{~mA}$ and $326 \mathrm{~mA}$. At $276 \mathrm{~mA}$ and below, all the bunches move closer, or further away in unison, according to fig. $3(\mathrm{~b})$. At $326 \mathrm{~mA}$ and above, the 
accordion oscillation changes according to fig. 3(c). The second bunch in the train oscillates in the opposite direction as the third and fourth bunch. These measurements, in conjunction with the spectrum analyzer results, reveal that the change in oscillation mode occurs between 276 and $326 \mathrm{~mA}$.

The dipole mode signal (and no signals indicating higher modes of oscillation) was the only signal observed by the spectrum analyzer during these measurements.
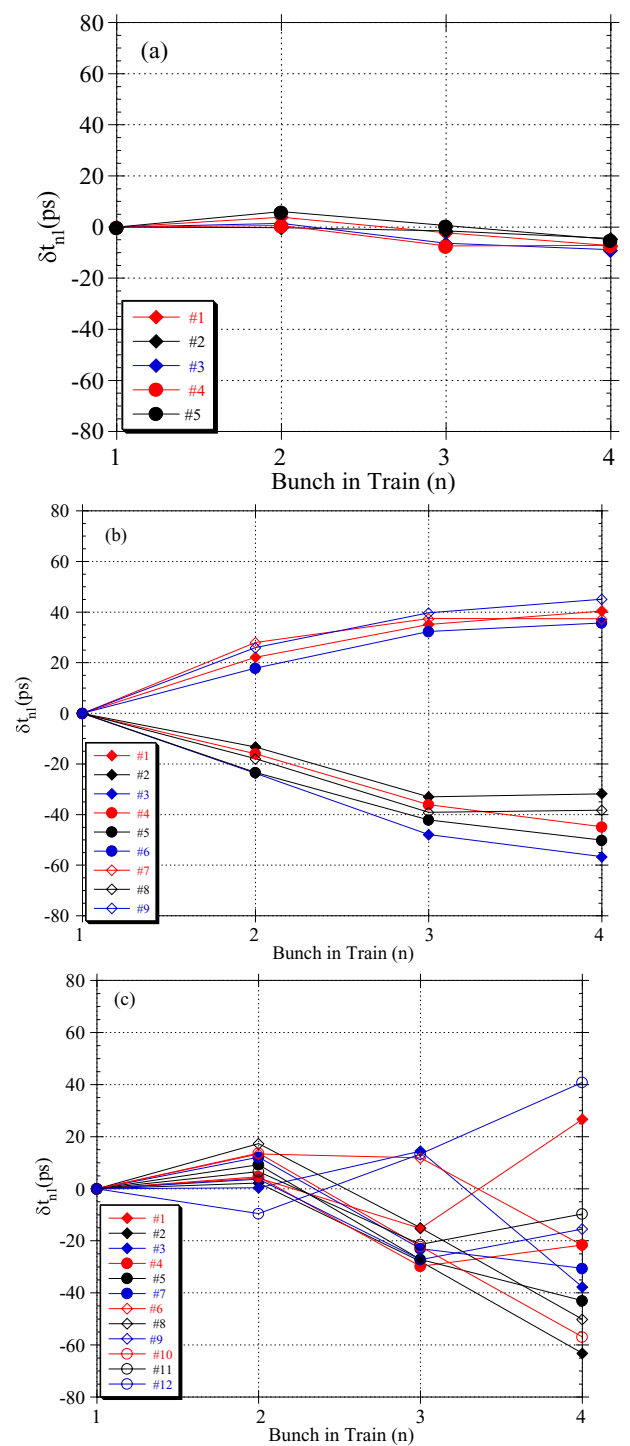

FIG. 3 (color). The time of arrival oscillation $\left(\delta \mathrm{t}_{\mathrm{n} 1}\right)$

when (a) the train is stable and the total beam current is $276 \mathrm{~mA}$, (b) the train is unstable and the total beam current is $276 \mathrm{~mA}$ and (c) the train is unstable and the total beam current is $326 \mathrm{~mA}$

When only normal conducting copper cavities were present in CESR, oscillations having the same spectral characteristics as longitudinal quadrupole-coupled bunch oscillations were detected by driving the dipole-mode to high amplitude[2]. The presence of these quadrupolemode-like oscillations drastically changes the bunch distribution and leads to beam loss. With only the dipole oscillations present, no change in the bunch distribution is expected.

\section{CONCLUSIONS}

In this paper, we report the results of detailed measurements of the longitudinal dipole-coupled bunch instability at CESR. The measurements were made using a dual-sweep synchroscan streak camera. The experiments have allowed us to understand the longitudinal dynamics of the instability during highenergy physics collisions. The instability is damped by a strong feedback system that reduces its affect on luminosity and allows an increase in current threshold.

For a single train of bunches, we have concluded that: 1) above the instability threshold, the longitudinal feedback system stabilizes the temporal oscillations to approximately 5ps. Without feedback, the temporal oscillations have peak amplitudes of approximately 100ps. These oscillations, called bunch oscillations, degrade the High Energy Physics luminosity. 2) The oscillation modes within a train changes as a function of current. Just above the instability, an "accordion" mode of oscillation has been observed. The accordion mode has all the bunches in a train either moving together, or apart, in unison. At higher currents more complicated modes of oscillation are present. 3) The instability is less severe with the superconducting RF cavities. Previously, the instability would produce shape oscillations at high current and this would lead to beam loss[2]. The superconducting cavities have made significant difference in the characteristics of the instability.

The operation of the dual sweep synchroscan streak camera is understood. The camera has provided detailed measurements of the longitudinal dynamics of the longitudinal dipole-coupled bunch instability that could not be measured by a normal streak camera. The camera has proved to be a valuable diagnostic tool for characterizing beam oscillations and longitudinal beam dynamics for the Cornell Electron-Positron Storage Ring.

\section{ACKNOWLEDGMENTS}

The authors would like to thank the operation staff at the Laboratory of Nuclear Studies at Cornell University. We would also like to thank Robert Meller, David Rice, and Sasha Temnykh for useful discussions about the dynamics observed.

\section{REFERENCES}

[1] Belomestnykh, S., et al., "Superconducting RF System for the CESR Luminosity Upgrade: Design, Status, Plans". SRF 960529-02.

[2] Holtzapple, R.L., et al., "Multiple Bunch Longitudinal Dynamics Measurements of the Cornell Electron-Positron Storage Ring ". Phys. Rev. ST Accel Beams 4, 014401, 2001 